\title{
Finite Element Modeling of Receding Contact Problem
}

\author{
Murat Yaylacı $^{a^{*}}$, Mehmet Çağrı Bayrak ${ }^{b}$, Mehmet Avcar $^{c}$ \\ ${ }^{a}$ Recep Tayyip Erdogan University, Department of Civil Engineering, 53100, Rize, Turkey \\ ${ }^{b, c}$, Suleyman Demirel University, Department of Civil Engineering, 3220, Isparta, Turkey \\ ${ }^{*}$ E-mail address: murat.yaylaci@erdogan.edu.tr ${ }^{\mathrm{a}}$, mcbayrak@ yahoo.com ${ }^{\mathrm{b}}$, $\underline{\text { mehmetavcar@ } \text { yahoo.com }^{\mathrm{c}}}$ \\ ORCID numbers of authors: \\ 0000-0003-0407-1685 ${ }^{a}$, 0000-0002-9995-7325 $5^{b}$,0000-0002-0689-0601
}

Received date: 14.11 .2019

Accepted date: 26.11 .2019

\begin{abstract}
In this study, the contact problem of an elastic layer resting on the rigid foundation is examined. For this aim, two-dimensional analysis has been performed using Finite Element Method (FEM) based software called ANSYS. In the solution of the problem, the mass forces of the layers are neglected and the thickness through z-axis direction is taken as a unit since the problem is examined for the plane state. The contact length between the punch and layer and the resulting contact stresses are obtained for various dimensionless parameters.
\end{abstract}

Keywords: Contact Mechanics, Plane contact problem, FEM analysis

\section{Introduction}

Contact problems have found wide application areas in engineering structures of practical importance. Foundations, road and airport superstructures, railways, fuel tanks, grain silos, cylindrical shafts and marbles are some of examples of these applications. Therefore, contact problems attracted the attentions of many scientists, and so open literature includes numerous analytical and numerical studies on contact problems. Hertz [1] presented an analytical solution for the contact problem of two elastic bodies with curved surfaces. Weitsman [2] reported an approximate solution for the radius of contact between an elastic plate and a semi-infinite elastic half space. Keer et al. [3] considered the smooth receding contact between an elastic layer and a half space when the two bodies arc pressed together. Ratwani and Erdogan [4] examined the plane contact problem for an elastic layer lying on an elastic half space. Nowell and Hills [5] studied the plane elastic contact between a thin strip and symmetric rollers. Chan and Tuba [6] described modified finite element method for solving problems of elastic bodies in contact. Francavilla and Zienkiewicz [7] proposed a simple procedure of obtaining flexibility matrices in terms of contact pressures at possible contact points of two bodies allows the frictionless 
contact pressures to be solved as a quasi-linear problem. Jing and Liao [8] examined an improved finite element scheme for elastic contact problems with friction is improved by introducing the concept of a contact node pair. Garrido et al. [9] applied boundary element method to the receding contact problems with friction. Garrido and Lorenzana [10] presented a new algorithm for the boundary element analysis of the two-dimensional contact problem between elastic solids involving large displacements. Yaylac1 et al. [11] dealt with the numerical analysis of the symmetric contact problem of two bonded layers resting on an elastic half plane compressed with a rigid punch using FEM based ANSYS and ABAQUS software.

In the present paper, two-dimensional analysis is performed using Finite Element Method (FEM) based software called ANSYS [12] for the contact problem of an elastic layer resting on the rigid foundation subjected to the concentrated load by a rigid punch. Several analyses are conducted for determining the contact length between the punch and layer and the resulting contact stresses for versus dimensionless parameters.

\section{The Analysis of the Problem}

Figure 1 shows the geometry of frictionless contact problem of the rigidly supported elastic layer, where $h$ is the height of the elastic layer and the concentrated load is applied via a rigid punch with the radius $\mathrm{R}$ to the upper surface of the elastic layer. The layer is in contact with punch in the interval $(-a,+a)$ and extends along the $x$ axis in the range $(-\infty,+\infty)$. Since the problem is symmetrical with respect to the y axis, the calculations are made in the range $(0,+$ $\infty)$. In the solution of the problem, the thickness is taken as a unit since the problem is examined for the plane state, as well as the mass forces and friction are neglected in the solution of the problem.

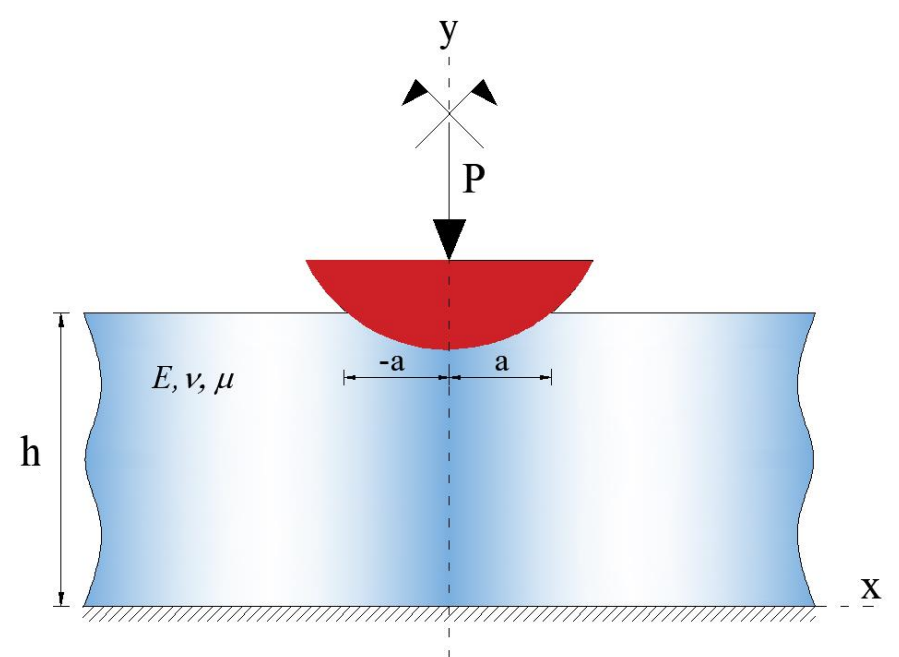

Fig. 1. The geometry of the problem

Here, $\mu, v$ and $E$ denote the shear modulus, Poisson's ratio, and Young's modulus of the layer, respectively.

Figure 2 shows the geometry of finite element analysis. The problem is modeled symmetrically with respect to the $y$ axis and the weight of the layer is neglected in here. Linear, elastic and isotropic materials are used in all parts of the finite element model. In the analyzes, the halflength and height of the layer taken to be $\mathrm{L}=1 \mathrm{~m}$ and $\mathrm{h}=0.1 \mathrm{~m}$, respectively. 


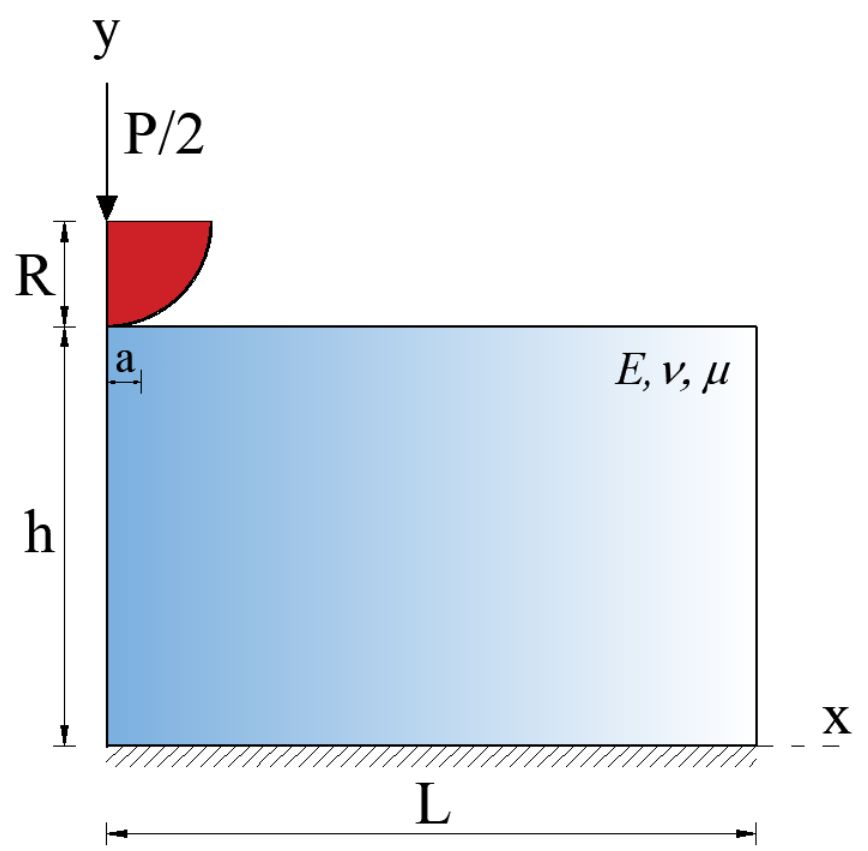

Fig. 2. The geometry of the analysis

Figure 3 shows the geometry of ANSYS. The element choice is especially important in determining the mathematical model to be used in the analysis. The elements are selected according to the type of analysis to be performed. Distinct types of elements may be used for static, thermal, fluid, or electromagnetic analyses. Similarly, whether the model to be analyzed is two or three dimensional is another significant factor in the element choice. Furthermore, the type and number of degrees of freedom of the joints of selected element are essential for the exact analysis. In the analysis, PLANE183 type structural element of the ANSYS package library is used. The PLANE183 type element is defined by eight joints and each joint has two degrees of freedom and no rotation as well as it has plasticity, withstands large flexes, and has a great deal of deformation.

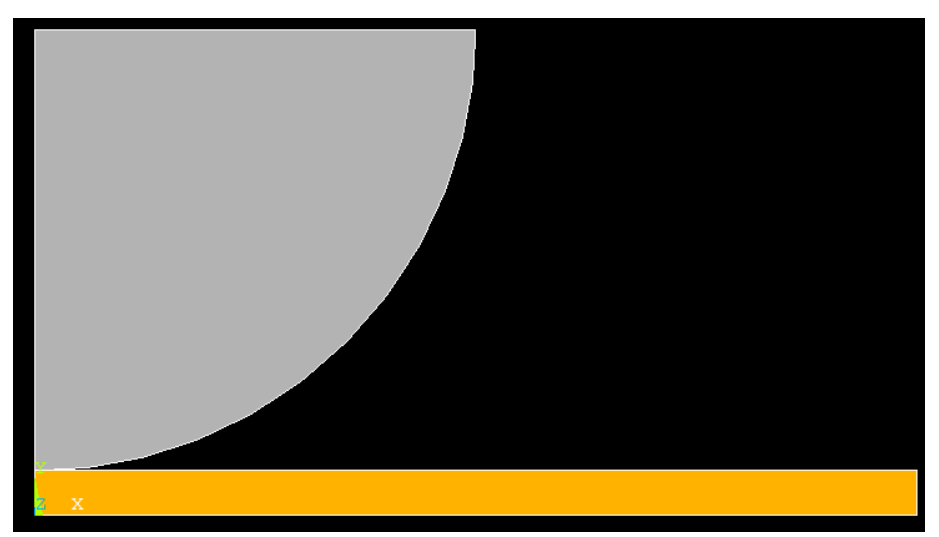

Fig. 3. The geometry of ANSYS

Figure 4 shows the contact pair of ANSYS. The surface-to-surface contact model is used to model the contact pair. Contact pairs consist of two element types, i.e., TARGET and CONTACT element types. The target surface TARGE169 and the contact surface CONTA172 with three joints are used to form the contact pair. 


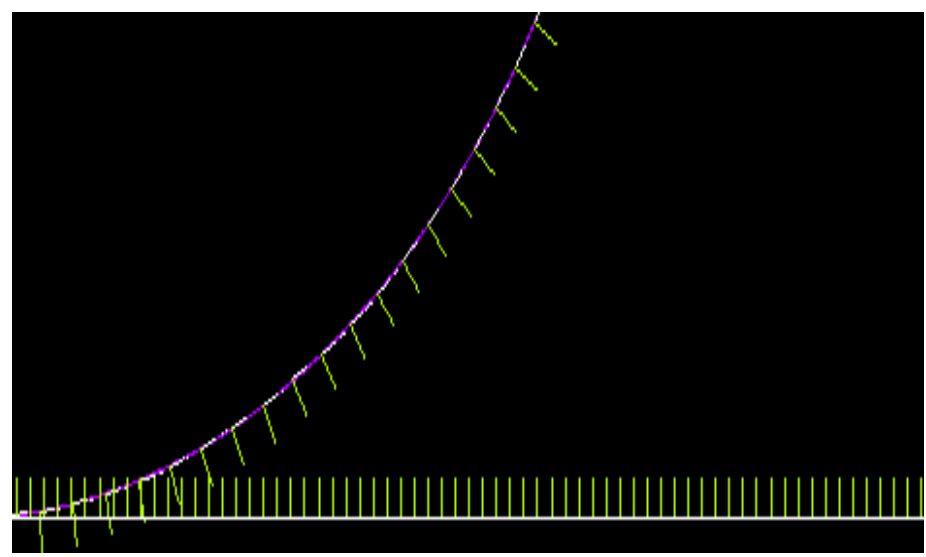

Fig. 4. The contact pair of ANSYS

Figure 5 shows the mesh of the present model. Here, the material properties and element types of the parts in the geometry are assigned mesh structure and size are determined. After the application of the boundary conditions and loading, the problem is solved with the help of the ANSYS program. 4155 joint points and 1450 elements are used to solve the present problem. The deformed shapes of the model are given in Figure 6.

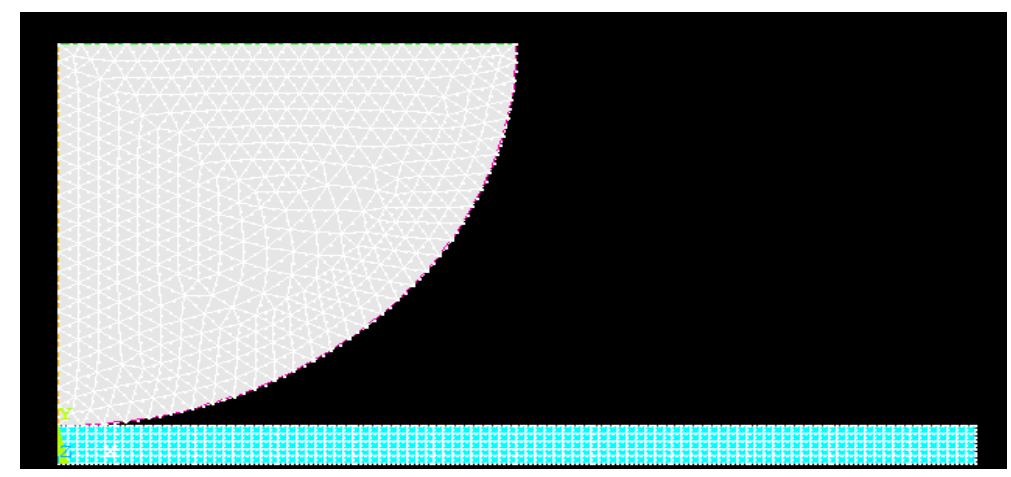

Fig. 5. The mesh of model
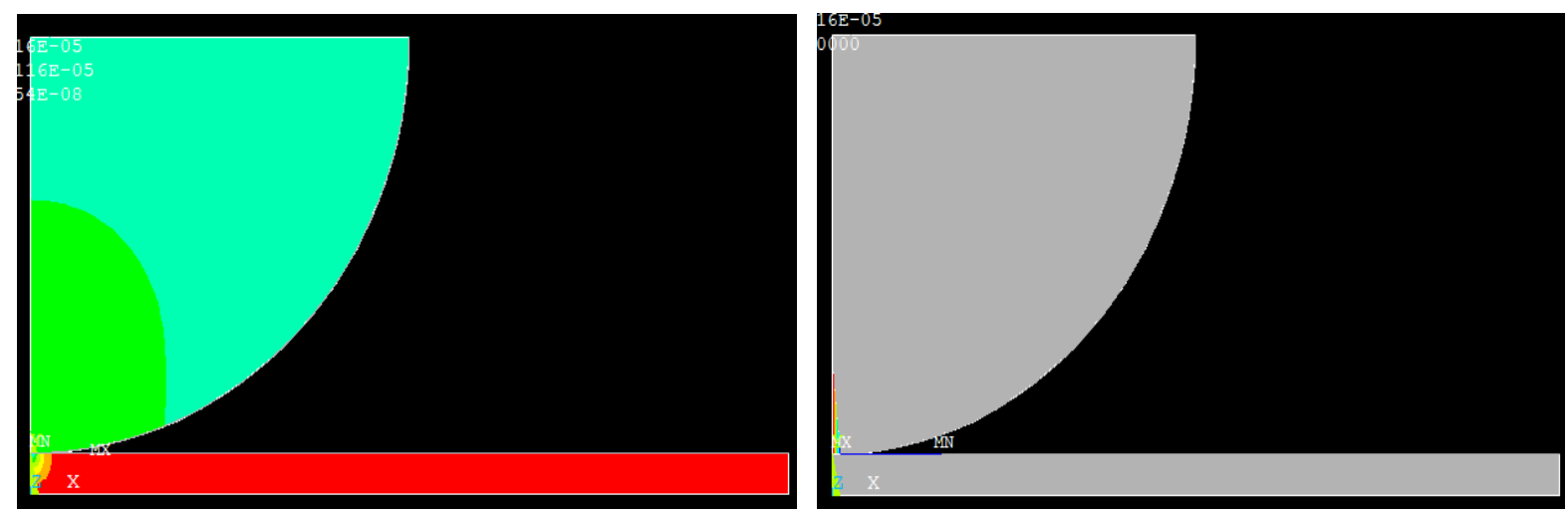

Fig. 6. The deformed shapes of the model 


\section{The Numerical Results}

Figure 7 shows the variation of contact length between the elastic layer and the rigid punch, $(\mathrm{a} / \mathrm{h})$, versus the punch radius and load ratio. Here, the variation of the load is expressed with the dimensionless ratio $\mu /(P / h)$. It is found that the contact distances decrease with the increase of the ratio $\mu_{0} /(P / h)$. Besides, the contact length increases with the increase of the punch radius.

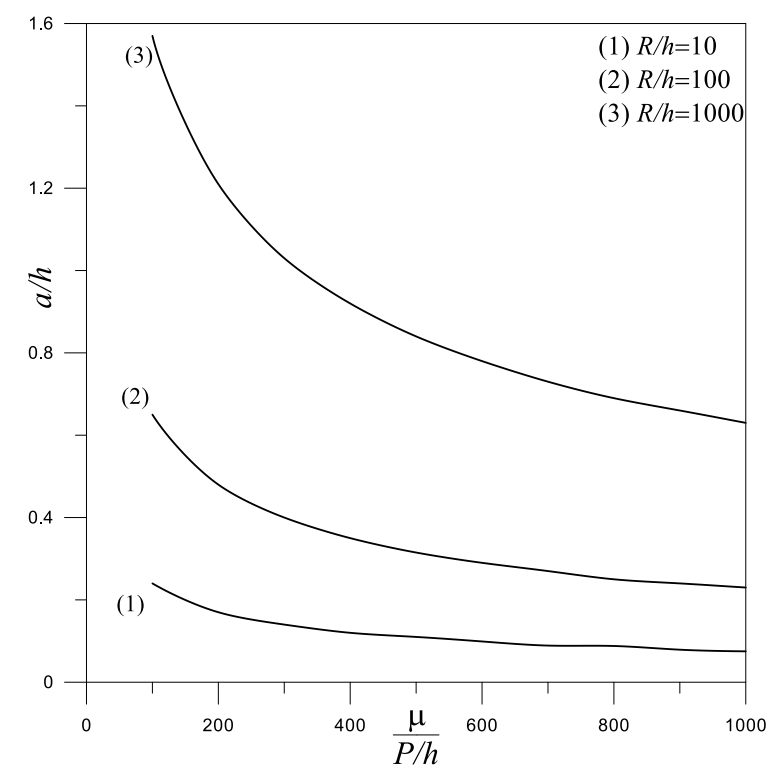

Fig. 7. The variation of the contact length versus the punch radius and load ratio $(v=0.25)$

Figure 8 shows the variation of the contact length versus punch radius and Poisson's ratio, $v$. It is seen that as Poisson's ratio increases, the contact length decreases, since the body becomes more rigid. Besides, the contact length increases with the increase of the punch radius.

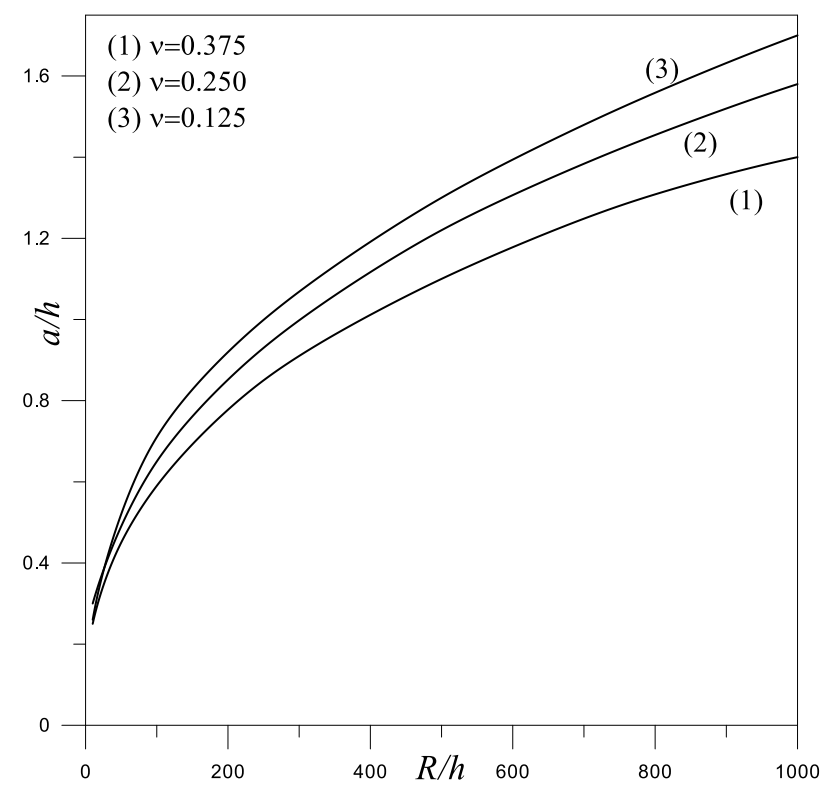

Fig. 8. The variation of the contact length versus the punch radius and Possions's ratio

$$
\left(\frac{\mu}{P / h}=100\right)
$$


Figure 9 shows the variation of dimensionless contact stress versus load ratios. It is concluded that as the load ratio increases, the dimensionless contact stresses under the punch increase because the decrease of half contact length.

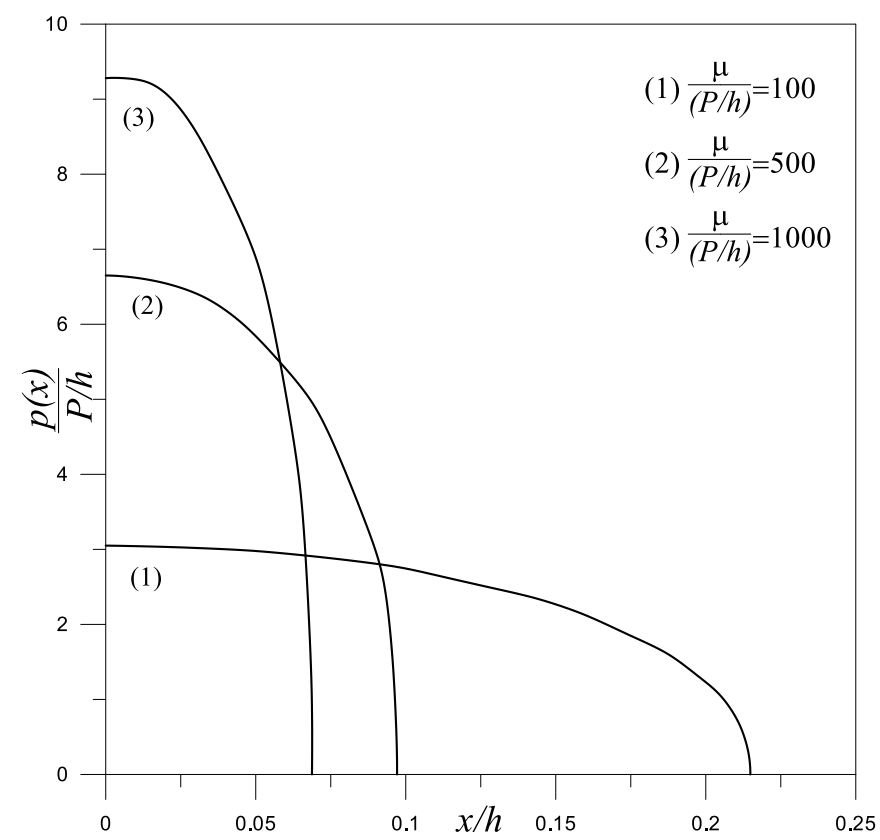

Fig. 9. The variation of dimensionless contact stress versus of load ratios $(v=0.25, R / h=10)$

Figure 10 shows the variation of the dimensionless contact stress versus punch radius. It is observed that as the block radius increases, the maximum value of the dimensionless contact stress under the punch decreases.

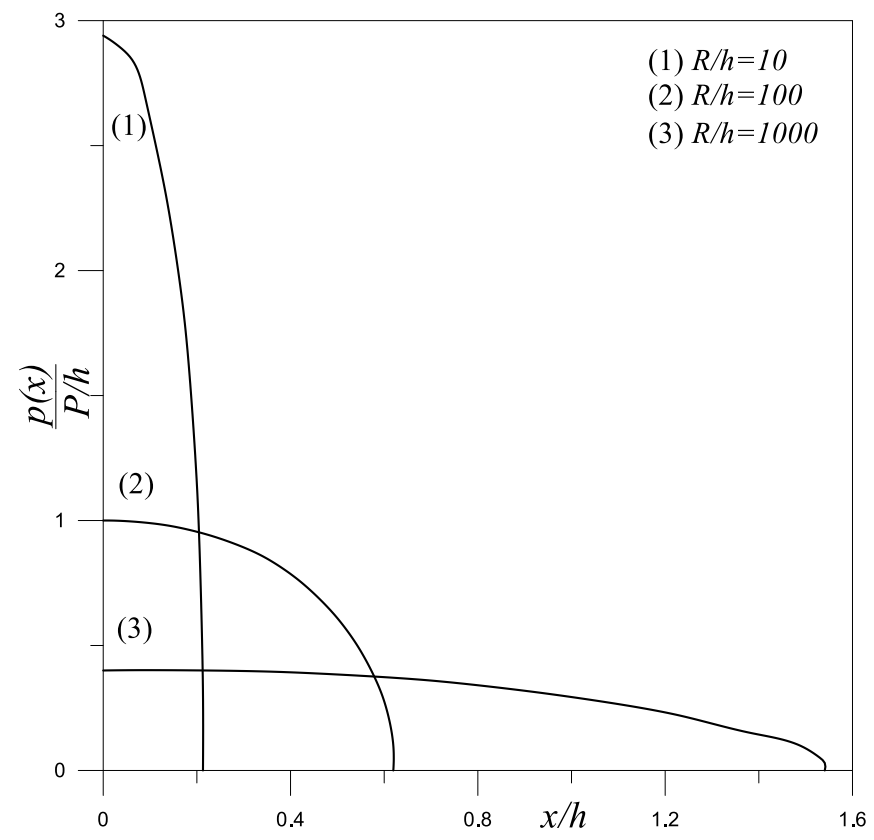

Fig. 10. The variation of the dimensionless contact stress versus punch radius $\left(v=0.25, \frac{\mu}{P / h}=\right.$ 100) 
Figure 11 shows the variation of the dimensionless contact stress versus Possion's ratio. It is found that as the value of Poisson's ratio decreases, the contact length increases and thus the load spreads over a larger area and the contact stresses decrease.

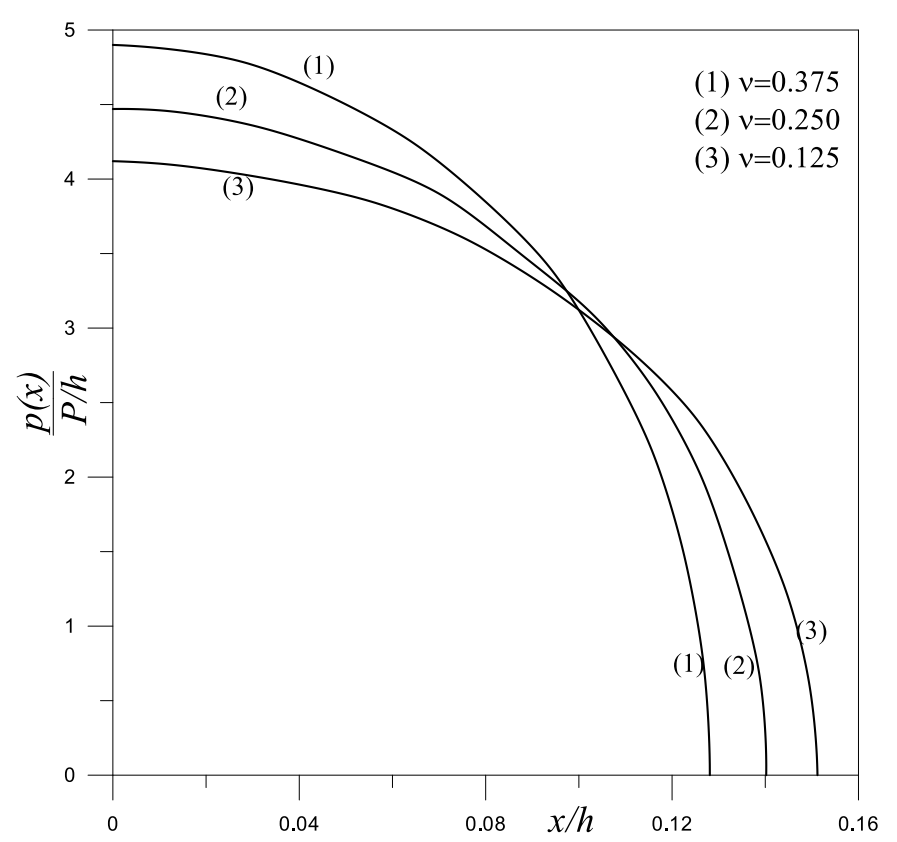

Fig. 11. The variation of the dimensionless contact stress versus Poisson's ratio $(R / h=10$,

$$
\left.\frac{\mu}{P / h}=100\right)
$$

\section{Conclusions}

In this study, contact problem of an elastic layer resting on the rigid foundation is examined using Finite Element Method (FEM) based software called ANSYS. The contact length between the punch and layer and the resulting contact stresses are obtained for various dimensionless parameters

Here, the following results are gotten

a) The contact distances decrease with the increase of the load ratio

b) The contact length increases with the increase of the punch radius

c) The dimensionless contact stresses under the punch increase with the increase of load ratio

d) The maximum value of the dimensionless contact stress decreases with the increase of the block radius

e) The contact stresses decrease with the decrease of the value of Poisson's ratio 


\section{References}

[1] Hertz, H., Ueber die Berührung fester elastischer Körper, Journal Fur Die Reine Und Angewandte Mathematik, 1882, 156-71, 1882. doi:10.1515/crll.1882.92.156

[2] Weitsman, Y., On the unbonded contact between plates and an elastic half space, Journal of Applied Mechanics, Transactions ASME, 1964. doi:10.1115/1.3564607

[3] Keer, L.M., Dundurs, J., Tsai, K.C., Problems involving a receding contact between a layer and a half space, Journal of Applied Mechanics, Transactions ASME, 1972. doi:10.1115/1.3422839

[4] Ratwani, M., Erdogan, F., On the plane contact problem for a frictionless elastic layer, International Journal of Solids and Structures, 1973. doi:10.1016/0020-7683(73)90021-8

[5] Nowell, D., Hills, D.A., Contact problems incorporating elastic layers, International Journal of Solids and Structures, 1988. doi:10.1016/0020-7683(88)90102-3

[6] Chan, S.K., Tuba, I.S., A finite element method for contact problems of solid bodies-Part I. Theory and validation, International Journal of Mechanical Sciences, 13, 615-25, 1971. doi:10.1016/0020-7403(71)90032-4

[7] Francavilla, A., Zienkiewicz, O.C., A note on numerical computation of elastic contact problems, International Journal for Numerical Methods in Engineering, 9, 913-24, 1975. doi:10.1002/nme.1620090410

[8] Jing, H.S., Liao, M.L., An improved finite element scheme for elastic contact problems with friction, Computers and Structures, 35, 571-8, 1990. doi:10.1016/00457949(90)90385-F

[9] Garrido, J.A., Foces, A., Paris, F., B.E.M. applied to receding contact problems with friction, Mathematical and Computer Modelling, 15, 143-53, 1991. doi:10.1016/08957177(91)90060-K

[10] Garrido, J.A., Lorenzana, A., Receding contact problem involving large displacements using the BEM, Engineering Analysis with Boundary Elements, 21, 295-303, 1998. doi:10.1016/s0955-7997(98)00018-6

[11] Yaylaci, M., Terzi, C., Avcar, M., Numerical analysis of the receding contact problem of two bonded layers resting on an elastic half plane, Structural Engineering and Mechanics, accepted, 2019

[12] ANSYS, Swanson Analysis Systems Inc. Houston PA, USA., 2013 